\title{
Correlation between the extraordinary Hall constant and electrical resistivity minima in Co-rich metallic glasses
}

\author{
A. K. Majumdar* and P. K. Khatua \\ Department of Physics, Indian Institute of Technology, Kanpur 208016, Uttar Pradesh, India \\ K. D. D. Rathnayaka and D. G. Naugle \\ Department of Physics, Texas A\&M University, College Station, Texas 77843-4242, USA \\ (Received 28 November 2003; revised manuscript received 1 March 2004; published 21 June 2004)
}

\begin{abstract}
The Hall effect has been studied in some Co-rich ferromagnetic metallic glasses which show resistivity $(\rho)$ minima at low temperatures. It is found that the extraordinary Hall constant $\left(R_{s}\right)$ shows a corresponding minimum. The scaling relation $R_{s} \sim \rho^{n}$ holds with $n \simeq 2$ showing the dominance of quantum transport in these high-resistive disordered systems. The temperature dependences of magnetization and electrical resistivity are also interpreted in terms of existing theories.
\end{abstract}

DOI: $10.1103 /$ PhysRevB.69.214417

PACS number(s): 72.15.Cz, 72.15.Rn, 75.30.Ds, 75.50.Kj

\section{INTRODUCTION}

The Hall resistivity $\left(\rho_{H}\right)$ in a crystalline ferromagnet is, for $T \ll T_{c}$, given by ${ }^{1}$

$$
\rho_{H}=R_{0} B+\mu_{0} R_{s} M_{s},
$$

where $B$ is the magnetic induction and $\mu_{0}$ is the permeability of free space. The Lorentz force acting on the charge carriers is responsible for the first term where $R_{0}$ is called the "ordinary Hall constant." This term is present in nonmagnetic materials as well. The second term, characteristic of a ferromagnet, depends on the saturation magnetization $M_{s}$ while $R_{s}$ is known as the "extraordinary" or the "spontaneous" Hall constant. In Eq. (1) $R_{0}$ and $R_{s}$ have the same units of $\Omega \mathrm{m} / \mathrm{T}$ in SI. Two different mechanisms are responsible for $R_{s}$.

\section{A. Nonclassical transport}

It has been shown ${ }^{1}$ that whenever the dimensionless parameter $\hbar / \tau E_{F}$, where $\tau$ is the electron relaxation time and $E_{F}$ is the Fermi energy, becomes large, the classical Boltzmann equation does not hold and nonclassical terms begin to dominate. Karplus and Luttinger ${ }^{2}$ and later on Luttinger, ${ }^{3}$ using a quantum transport theory, were the first to predict that $R_{s}$ is proportional to $\rho^{2}$, where $\rho$ is the electrical resistivity. Due to the spin-orbit interaction present in a ferromagnet, the symmetry of the problem is low and there is a nonzero transverse electric field which gives rise to a Hall voltage. The above theories are quite complicated and involved. However, later on Berger ${ }^{4}$ gave an intuitive picture of the physical mechanisms responsible for the Hall effect in concentrated ferromagnetic alloys and high temperatures. He proposed that an electron undergoes a discontinuous and finite "side jump" at every scattering by impurities or phonons. This also leads to the equation

$$
R_{S}=b \rho^{2} \text {. }
$$

Both approaches, ${ }^{3,4}$ by the way, predict that $b$ in Eq. (2) is independent of the nature of the scatterer.

\section{B. Asymmetric scattering}

In the case of very dilute alloys at low temperatures the Boltzmann equation should be adequate. Here $R_{S}$ is caused by "asymmetric scattering" of magnetized conduction electrons as proposed by Smit. ${ }^{5}$ In the presence of a spin-orbit interaction there is a left-right asymmetry in the differential scattering cross-section about the $\vec{J}-\vec{M}$ plane where $\vec{J}$ is the current density. As a result charge carriers tend to pile up on one side of the sample producing a transverse electric field. Here

$$
R_{s}=a \rho .
$$

The Hall effect in ferromagnetic metallic glasses has been reviewed at length by McGuire, Gambino, and O'handley ${ }^{6}$ and Egami. ${ }^{7}$ Equation (2) is found to be valid for $R_{S}$ in Fe, $\mathrm{Ni}$, and Co-based metallic glasses indicating the expected dominance of the side-jump mechanism in these highresistive metallic glasses. The values of $R_{s}$ in amorphous metals and alloys are, in general, much larger than those of their crystalline counterparts. This is a direct consequence of the much higher resistivity in the amorphous state. Also, here $R_{s}$ has a weak temperature dependence because of the corresponding weak temperature dependence of the electrical resistivity since the disorder-induced resistivity dominates over the thermal contribution. Lachowicz ${ }^{8}$ interpreted $R_{S}$ in amorphous GdCo sputtered films in terms of two contributions, one coming from the skew scattering and the other from the nonclassical side-jump mechanism. However, Shiba et al. ${ }^{9}$ explained $R_{s}$ in amorphous Co-metal film by only the quantum mechanical side-jump mechanism $\left(R_{s} \sim \rho^{2}\right)$. From the temperature dependence of both $R_{s}$ and $\rho$, Ivkov et al. ${ }^{10}$ obtained $R_{S} \sim \rho^{2}$ in a vast majority of $\mathrm{Fe} / \mathrm{Co} / \mathrm{Ni}$-based amorphous alloys. Also, $R_{s}$ is found to be positive for $\mathrm{Fe}$ and $\mathrm{Co}$ and negative for Ni-containing metallic glasses ${ }^{1}$ at $300 \mathrm{~K}$. However, most of the work reported till now is on those amorphous alloys where $\rho$ monotonically increases with temperature and as does $R_{s}$. The motivation behind the present work is to track the scaling law $R_{s} \sim \rho^{n}$ where $\rho$ shows a minimum at $T m$, i.e., does $R_{s}$ also show a minimum? How- 
ever, we find that if $R_{s} M_{s}$ decreases with temperature (as in the present case, but not necessarily so for other materials) by the same amount as $M_{s}$ decreases with temperature, the change of $R_{s}$ with temperature is very small (specially around the minimum) and becomes comparable to its experimental error. As a result a weak minimum is found in $R_{s}$ near $T m$ in only two of the alloys. Also, we find that $n \simeq 2$ (sidejump mechanism) showing the dominance of the nonclassical mechanism for the origin of $R_{s}$.

\section{EXPERIMENTAL DETAILS}

The as-received metallic glass samples are in the form of ribbons, of typical width $\sim 1 \mathrm{~mm}$ and thickness $\sim 0.030 \mathrm{~mm}$. The nominal compositions of the amorphous alloys are as follows.

(i) Sample 1: $\mathrm{Fe}_{5} \mathrm{Co}_{50} \mathrm{Ni}_{17} \mathrm{~B}_{16} \mathrm{Si}_{12}$.

(ii) Sample 2: $\mathrm{Fe}_{7.8} \mathrm{Co}_{31.2} \mathrm{Ni}_{34} \mathrm{Cr}_{5} \mathrm{~B}_{14} \mathrm{Si}_{8}$.

(iii) Sample 3: $\mathrm{Fe}_{7.8} \mathrm{Co}_{31.2} \mathrm{Ni}_{29} \mathrm{Cr}_{10} \mathrm{~B}_{14} \mathrm{Si}_{8}$.

(iv) Sample 4: $\mathrm{Fe}_{7.8} \mathrm{Co}_{31.2} \mathrm{Ni}_{24} \mathrm{Mn}_{15} \mathrm{~B}_{14} \mathrm{Si}_{8}$.

They were prepared by melt quenching. The amorphous nature of the samples was checked by X-ray diffraction measurements using a Rich and Seifert Isodebyeflex 2002 diffractometer with a $\mathrm{Cu}$ target $\left(\mathrm{K}_{\alpha}=1.54 \AA\right)$. The results show broad peaks at low angles due to short-range order. There was no observable crystalline phase. The Hall resistivity $\left(\rho_{H}\right)$ was measured using a standard 5-probe dc method for minimizing the resistive voltage arising from the misalignment of the Hall voltage probes. A voltage divider was made for each sample using two General Radio decade resistance boxes. The misalignment voltage was adjusted to within $1 \mu \mathrm{V}$ or better at each temperature. The Hall resistivity was measured at 27 temperatures between 6 and $190 \mathrm{~K}$ and magnetic fields up to $3 \mathrm{~T}$. The electrical resistivity in zero field was measured using a standard 4-probe dc setup from 4.2 to $200 \mathrm{~K}$ at every $1 \mathrm{~K}$. The magnetization was measured in a magnetic field $\mu_{0} H=1 \mathrm{~T}$ using a Quantum Design superconducting quantum interference device (SQUID) magnetometer. $T_{c}$ 's of samples 1, 2, 3, and 4 are found to be $\simeq 395,386,222$, and $368 \mathrm{~K}$, respectively.

\section{RESULTS AND DISCUSSION}

\section{A. Hall effect}

Figure 1 shows the Hall resistivity $\left(\rho_{H}\right)$ against magnetic induction $B$ for sample 4 at temperatures of $6,49,90,131$, and $188 \mathrm{~K}$. In the Hall geometry the magnetic field is perpendicular to the sample plane and so the demagnetization factor $N \simeq 1$. Thus $\vec{B}=\mu_{0}\left[\vec{H}_{\text {applied }}+(1-N) \vec{M}_{s}\right] \simeq \mu_{0} \vec{H}_{\text {applied }}$. $\rho_{H}$ shows the typical behavior of a ferromagnet for $T \ll T_{c}$ $=368 \mathrm{~K}$ where $\rho_{H}$ almost saturates beyond a certain $B$ (here $1 \mathrm{~T})$ and varies linearly with a slope $R_{0}$ and an intercept $\mu_{0} R_{s} M_{s} . R_{0}$ vs $T$ for all the samples is plotted in Fig. 2 up to $190 \mathrm{~K}$. The solid lines are just guides to the eye. $R_{0}$ is found to be positive over most of the temperature range and has a weak temperature dependence (except for sample 3 ) indicating minimal changes in the band structure in this temperature range. A sign change from negative to positive is observed below $30 \mathrm{~K} . R_{0}$ has a much stronger temperature dependence

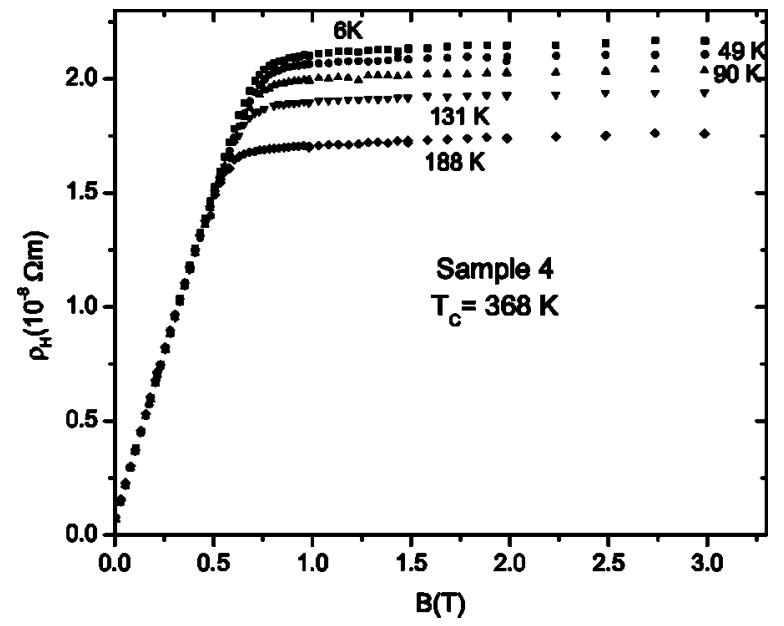

FIG. 1. Hall resistivity $\rho_{H}$ vs magnetic induction $B$ for sample 4 at $6,49,90,131$, and $188 \mathrm{~K}$. They show typical behavior of a ferromagnet above $1 \mathrm{~T}$.

for sample 3. Whereas for the other three samples $190 \mathrm{~K}$ $\simeq T_{c} / 2$, it is $\simeq T_{c}$ for sample 3 . For ferromagnets the band splitting due to the exchange interaction disappears around $T_{c}$. This affects the carrier density as well as their conductivities making $R_{0}$ strongly temperature dependent around $T_{c}$ as is the case with sample 3 .

$\mu_{0} R_{s} M_{s}$ is plotted against temperature in Fig. 3 for all the samples. They show a weak decrease with temperature for $T<50 \mathrm{~K}$. The solid lines are just guides to the eye. As expected for sample $3, R_{s} M_{s} \rightarrow 0$ as $T \rightarrow T_{c}(\simeq 222 \mathrm{~K})$. However, to extract $R_{s}(T)$ we use the SQUID data for $M$ vs $T$ which is shown in Fig. 4 at an external field of $1 \mathrm{~T}$. Comparing Figs. 3 and 4 we observe that from 5 to $190 \mathrm{~K}, R_{s} M_{s}$ decreases by $11.6,26.7,67.4$, and $19.3 \%$ and $M_{s}$ by 15.7 , 26.7, 64.6, and $22.4 \%$ for samples $1,2,3$, and 4 , respectively. This results in a change of only $4,0,3$, and $3 \%$, respectively, for $R_{s}$ with $T$ over this wide range of temperature. Thus to find the temperature dependence of $R_{s}$ and ultimately to check the scaling relation $R_{S} \sim \rho^{n}$ becomes ex-

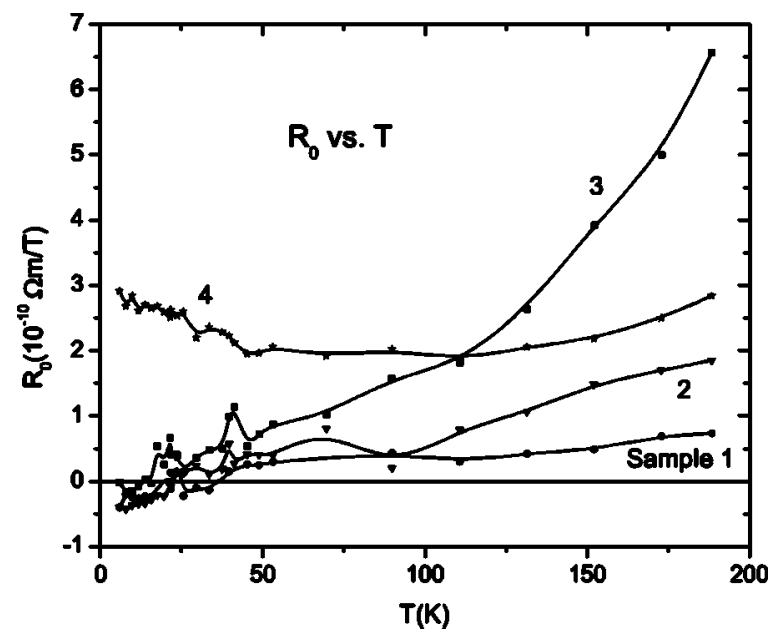

FIG. 2. The ordinary Hall constant $R_{0}$ vs temperature for all the samples showing a weak temperature dependence except for sample 3. The solid lines are just guides to the eye. 


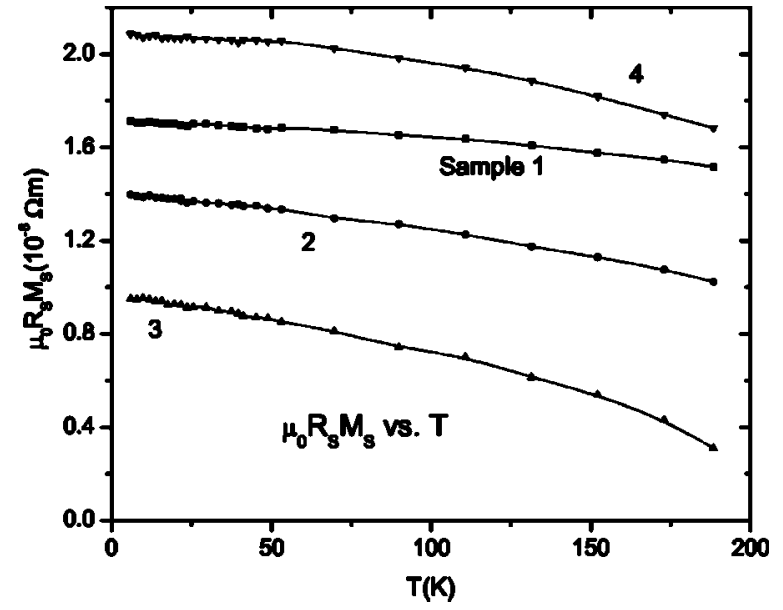

FIG. 3. $\mu_{0} R_{s} M_{s}$ (intercept with the $\rho_{H}$ axis of Fig. 1) vs temperature for all the samples showing a weak temperature dependence below $50 \mathrm{~K}$. The solid lines are just guides to the eye.

tremely difficult, if not impossible for some of the present samples. This is because of the very similar decrease of $R_{s} M_{s}$ and $M_{s}$ with temperature. For example in sample $2, R_{s}$ hardly changes $(0 \%)$. For sample 3 , although $R_{s}$ apparently decreases by $3 \%$, the accuracy of $R_{s}(T)$ is very poor since it is determined from the large decrease of $R_{s} M_{s}(67.4 \%)$ and $M_{s}$ (64.6\%) with $T$ due to its $T_{c}$ of only $222 \mathrm{~K}$. This decrease of $3 \%$ in $R_{s}$ is not significant since the errors in $R_{s} M_{s}$ and $M_{s}$ are at least $1 \%$ each.

The temperature dependence of the electrical resistivity $(\rho)$ is shown in Fig. 5 for all the samples. The data for samples 1,2 , and 3 are taken every $1 \mathrm{~K}$, and thus the curves look almost continuous. They show resistivity minima $\left(T_{m}\right)$ at $15,65,35$, and $24 \mathrm{~K}$, respectively, for samples 1,23 , and 4. In the temperature range of 5-190 K (Fig. 5) $\rho$ has a weak temperature dependence for samples 2 and 3 and somewhat stronger for 4 and 1 . In order to correlate $R_{s}$ with $\rho$ we have plotted in Figs. 6 and 7 their $\left(R_{s}\right.$ and $\left.\rho\right)$ temperature depen-

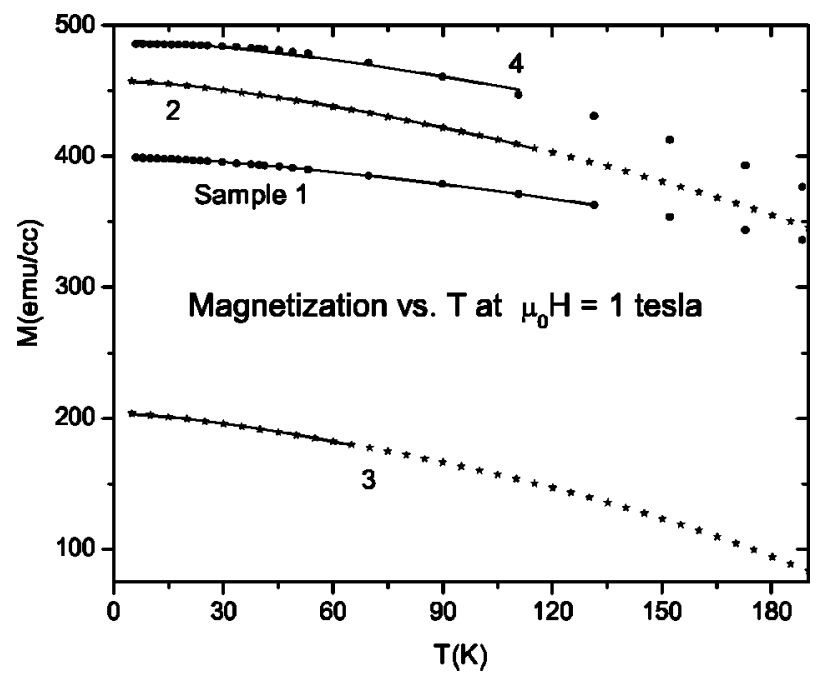

FIG. 4. Magnetization $M$ vs temperature for all the samples at $\mu_{0} H=1 \mathrm{~T}$. The solid lines are the best fits of $M$ to Eq. (4) up to $T$ $\simeq 0.3 T_{c}$.

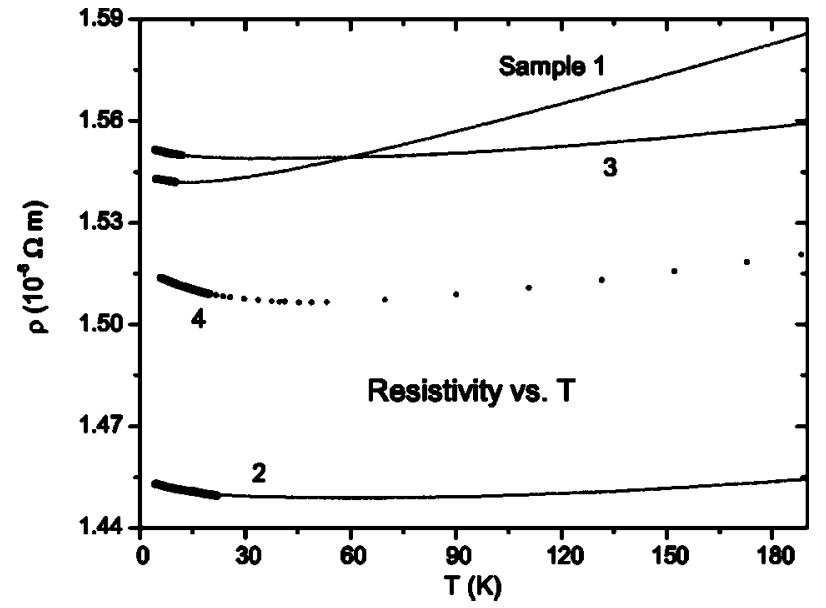

FIG. 5. Electrical resistivity $\rho$ vs temperature for all the samples. The points are the raw data, and the thick solid lines are the best fits of $\rho$ to Eq. (6) at very low temperatures $(T \leqslant 22 \mathrm{~K})$.

dences on the same graph for the more favorable samples 1 and 4 , respectively [which show meaningfully measurable $\approx(3-4) \%$ changes in $R_{S}(T)$ ]. It is amply clear from Fig. 6 that sample 4 shows a minimum in $R_{s}$ at about $25 \mathrm{~K}$, the same temperature at which the electrical resistivity shows a minimum. The large error bars in $R_{s}$ are due to the similar decrease of $R_{s} M_{s}$ and $M_{s}$ with $T$, as mentioned above. The minimum is not as clear in Fig. 7 (sample 1) but it cannot be overlooked. We must mention here that $R_{s}(T)$ has to be determined from two independent measurements, the Hall resistivity and the magnetization. Also, in ferromagnetic materials where $R_{s} M_{s}$ increases with temperature the extraction of $R_{s}(T)$ is much more accurate since $M_{s}$ always decreases with temperature. A comparison of the absolute values of $R_{s}$ in our samples (typically $3 \times 10^{-8} \Omega \mathrm{m} / \mathrm{T}$ ) gives a reasonable agreement with the values obtained by Ivkov et al. ${ }^{10}$ (typically $\left.2 \times 10^{-8} \Omega \mathrm{m} / \mathrm{T}\right)$. Also, $R_{s}$ increases from $2.4 \times 10^{-8}$ to $4 \times 10^{-8} \Omega \mathrm{m} / \mathrm{T}$ from sample 2 to 3 which have similar com-

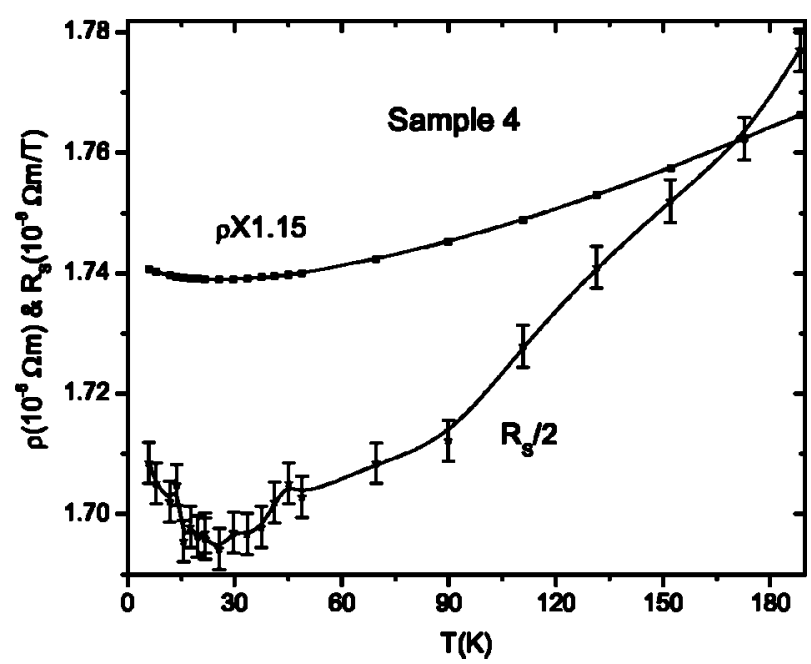

FIG. 6. $R_{s}$ and $\rho$ vs $T$ for sample 4. Both show a minimum around $25 \mathrm{~K}$. The error bars in $R_{s}$ are also shown. The solid lines are just guides to the eye. 


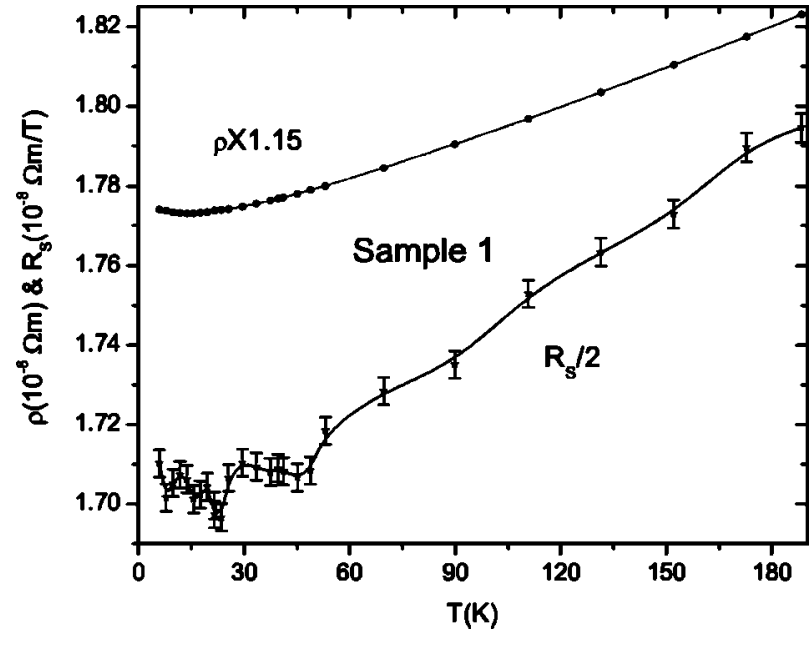

FIG. 7. The same as in Fig. 6 except that they are now for sample 1 .

position but differ in $\mathrm{Cr}$ by only $5 \%$ at the cost of Ni. Since $R_{S}$ in Ni is negative, ${ }^{1}$ less Ni makes $R_{S}$ more positive in these Co-rich $\left(R_{s}>0\right)$ metallic glasses.

To summarize, Figs. 6 and 7 clearly show that $R_{s}$ and $\rho$ go hand in hand. To the best of our knowledge there is no theoretical work on the effect of the electron-electron interaction on the side-jump mechanism or any other quantum transport theory for $R_{s}$. The coincidence of the minima only implies that the competing mechanisms responsible for the resistivity minima (viz., $\rho$ decreasing with temperature due to $e-e$ interaction and increasing with temperature due to electron-phonon scattering) also guide the temperature dependence of $R_{s}$ through $\rho$. For samples 2 and 3, there is negligible temperature dependence of $R_{s}$, and no correlation with $\rho$, which also shows a very weak temperature dependence. To find the exponent $n$ of $R_{s} \sim \rho^{n}$ we have plotted in Fig. $8 \ln R_{s}$ vs $\ln \rho$ for both samples 1 and 4 . The slope gives $n=2.2 \pm 0.1$. In these high resistivity $(\sim 150 \mu \Omega \mathrm{cm})$ metal-

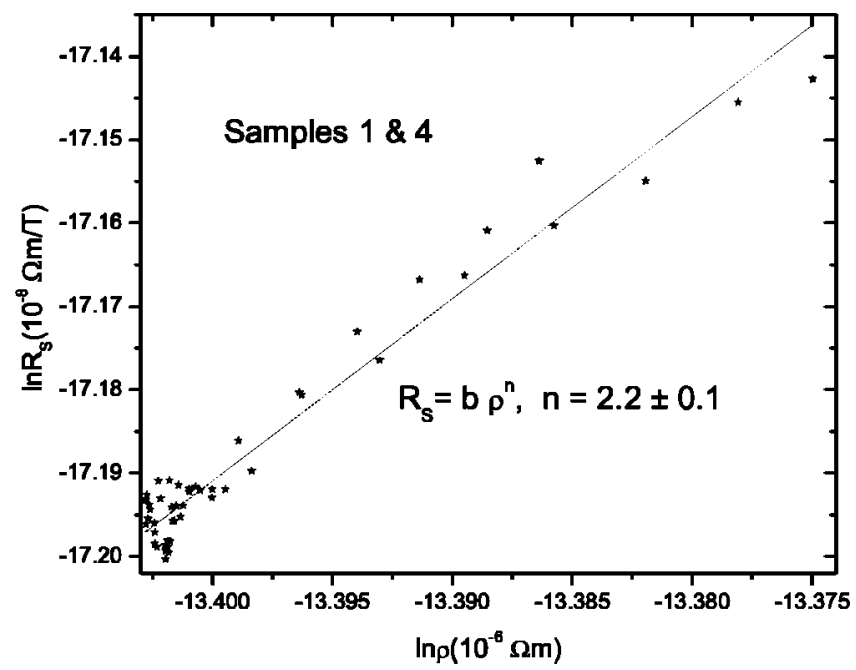

FIG. 8. $\ln R_{s}$ vs $\ln \rho$ for samples 1 and 4 on the same plot. The straight line is the best fit to the equation $R_{s}=b \rho^{n}$ with $n$ $=2.2 \pm 0.1$. lic glasses nonclassical transport clearly dominates over the Smit asymmetric scattering ${ }^{5}$ for which $n=1$.

\section{B. Magnetization}

The good quality of magnetization data (Fig. 4) permits analysis of the process of thermal demagnetization in these metallic glasses. In both crystalline and amorphous ferromagnets, consideration of only the harmonic term in the spin-wave dispersion relation gives the following expression for the magnetization: ${ }^{11,12}$

$$
M(T)=M(0)\left[1+A T^{3 / 2}\right] .
$$

Many amorphous ferromagnets follow Eq. (4) till $T$ $\cong 0.3 T_{c}{ }^{13}$

The spin-wave stiffness constant is related to $A$ above and is given by

$$
D=\frac{k_{B}}{4 \pi}\left(\frac{2.612 g \mu B}{M(0) A}\right)^{2 / 3} .
$$

The $M(T)$ data (Fig. 4) have been fitted to Eq. (4) up to $T$ $\simeq 0.3 T_{c}$. The solid lines in Fig. 5 represent the best fits to Eq. (4). These fits are quite good with correlation coefficients $\simeq 0.999$ and $\chi^{2}$ consistent with the experimental resolution. The spin-wave stiffness constants, calculated from Eq. (5), are found to be $108.6,75.8,71.2$, and $89.9 \mathrm{meV}^{2}$ for samples $1,2,3$, and 4 , respectively. These values are comparable to the value $100 \mathrm{meV} \AA^{2}$ found in many Fe-B metallic glasses. ${ }^{14} M(0)$ in samples 2 and 3 (which are very similar in composition but there is $5 \%$ more $\mathrm{Cr}$ in sample 3 ) are 460 and $200 \mathrm{emu} / \mathrm{cc}$, respectively. $\mathrm{Cr}-\mathrm{Cr}$ interaction in the localized model is antiferromagnetic and hence just $5 \%$ more $\mathrm{Cr}$ in sample 3 understandably reduces $M(0)$ considerably by a factor of $\sim 2$. A similar interesting consequence of antiferromagnetism of $\mathrm{Cr}$ is the reduction in $T_{c}$ from 386 to $222 \mathrm{~K}$.

\section{Electrical resistivity}

The relative accuracy of the electrical resistivity data shown in Fig. 5 is 10 ppm. Their values vary between 145 and $155 \mu \Omega \mathrm{cm}$ and have no significant composition dependence except that an additional $5 \% \mathrm{Cr}$ in sample 3 (over sample 2) increases $\rho$ from 145 to $155 \mu \Omega \mathrm{cm}$. Cr increases $\rho$ significantly also in crystalline $\mathrm{NiCr}$ binary alloys. ${ }^{13}$ Many of the metallic glasses which have large electrical resistivity show minima in their temperature dependence similar to those shown in Fig. 5. They can be explained by quantum interference effects. ${ }^{15,16}$ The equation

$$
\rho(T)=\rho_{0}-A T^{1 / 2}
$$

describes this resistivity data very well below $T_{m}$ with $A$ $=(400-1000) \Omega \mathrm{m} \mathrm{K}^{-1 / 2}$ in agreement with many other metallic glass samples. ${ }^{17,18}$ The thick solid lines in Fig. 5 are the best fits to Eq. (6) and yield correlation coefficients of $\simeq 0.99$. Electron-electron interaction effects, in the presence of weak localization, predict $\rho \sim \sqrt{T}$ well below $T_{m}$. Thus the fits extended only to $12,22,10$, and $20 \mathrm{~K}$, respectively, for samples $1,2,3$, and 4 . 


\section{CONCLUSIONS}

The minima in the electrical resistivity and the extraordinary Hall effect go hand in hand in two of the ferromagnetic metallic glasses having a high resistivity $\simeq 150 \mu \Omega \mathrm{cm}$. In the other two samples the variation of $R_{s}$ with temperature is comparable to its error. This is due to the fact that $R_{s} M_{s}$ and $M_{s}$ have very similar decreases with increasing temperature. The temperature dependence of magnetization is well described by Bloch's $T^{3 / 2}$ law giving reasonable values of spinwave stiffness constants. The resistivity minima are ex- plained in terms of electron-electron interaction in the presence of weak localization as observed in many disordered systems.

\section{ACKNOWLEDGMENT}

The work was partially supported by the National Science Foundation (Grant Nos. INT-9603137 and 9602975). Work at Texas A\&M was also supported by the Robert A. Welch Foundation, Houston, TX (Grant No. A-0514).
*Author to whom correspondence should be addressed. Electronic address: akm@iitk.ac.in

${ }^{1}$ L. Berger and G. Bergmann, in Hall Effect and its applications, edited by C. L. Chien and C. R. Westgate (Plenum, New York, $1980)$, p. 55, and references therein.

${ }^{2}$ R. Karplus and J. M. Luttinger, Phys. Rev. 95, 1154 (1954).

${ }^{3}$ J. M. Luttinger, Phys. Rev. 112, 739 (1958).

${ }^{4}$ L. Berger, Phys. Rev. B 2, 4559 (1970).

${ }^{5}$ J. Smit, Physica (Amsterdam) 21, 877 (1955); 24, 39 (1958).

${ }^{6}$ T. R. McGuire, R. J. Gambino, and R. C. O'handley, in Ref. 1, p. 137.

${ }^{7}$ T. Egami, Rep. Prog. Phys. 47, 1601 (1984).

${ }^{8}$ H. K. Lachowicz, Phys. Status Solidi A 67, K131 (1981).

${ }^{9}$ K. Shiba, S. Tsunashima, S. Uchiyama, and S. Yoshino, IEEE Trans. Magn. 22, 1986 (1986).
${ }^{10}$ J. Ivkov, E. Babic, and Z. Markovic, J. Phys. Colloq. 49, 1301 (1988).

${ }^{11}$ F. Keffer, in Handbuch der Physik, edited by S. Flügge and H. P. J. Wijn (Springer, Berlin, 1966), Vol. XVIII/2, p. 1.

${ }^{12}$ U. Krey, Z. Phys. B 31, 247 (1978).

${ }^{13}$ F. E. Luborsky, in Ferromagnetic Materials, edited by E. P. Wohlfarth (North-Holland, Amsterdam, 1980), Vol. 1, p. 497.

${ }^{14}$ A. K. Majumdar, V. Oestreich, D. Weschenfelder, and F. E. Luborsky, Phys. Rev. B 27, 5618 (1983).

${ }^{15}$ P. A. Lee and T. V. Ramakrishnan, Rev. Mod. Phys. 57, 287 (1985).

${ }^{16}$ J. S. Dugdale, Contemp. Phys. 28, 547 (1987).

${ }^{17}$ R. W. Cochrane and J. O. Strom-Olsen, Phys. Rev. B 29, 1088 (1984).

${ }^{18}$ T. K. Nath and A. K. Majumdar, Phys. Rev. B 55, 5554 (1997). 